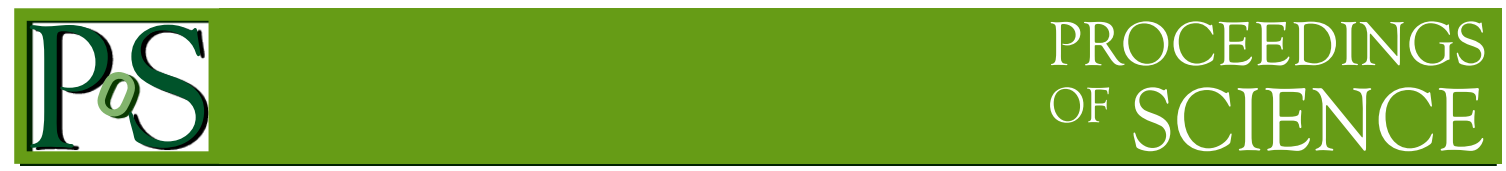

\title{
Old and New from SETI: a Review
}

\author{
Iván Almár ${ }^{1}$ \\ Konkoly Observatory of the Hungarian Academy of Sciences, \\ H-1121 Konkoly Thege Miklós út 15, Budapest, Hungary \\ E-mail: almarekonkoly.hu
}

This brief review of 55 years of SETI (Search for Extraterrestrial Intelligence) is intended to emphasize the recent paradigm change both in search strategy and in philosophy. The almost monopolistic situation of microwave radio searches after interstellar messages (old SETI) gave place to a much more diversified variety of methods and goals (new SETI). There is no positive result yet, but the tendency is promising

Keywords: SETI, METI, technomarkers, fundamentalist skepticism, instrumentalist skepticism

Frontier Research in Astrophysics

26-31 May, 2014

Mondello (Palermo), Italy

${ }^{1}$ Speaker

(C) Copyright owned by the author(s) under the terms of the Creative Commons 
Introduction

For almost half a century astrobiology and SETI were two distinct disciplines with different methods and different search strategies. Astrobiology concentrated on in situ search after extraterrestrial life in our Solar System (Mars in particular), as well as theoretical and laboratory studies of the origin of life. Since there is obviously no other technical civilization in the Solar System, than the terrestrial, SETI concentrated on search in the microwave by radio telescopes after messages or signals originating from inhabited planets around far away stars. Astrobiology or bioastronomy received financial support from governmental and university sources, because the eventual discovery of extraterrestrial life was and is considered an important and popular goal of solar system exploration. SETI was and is compromised by the UFO mythos and as a consequence was prohibited of governmental funding through NASA in 1993. Its limited continuity was guaranteed by private and university funds supported by its broad popularity in public opinion and in the media.

The situation has changed recently as a consequence of the discovery of almost one thousand extrasolar planets - making even a detailed study of their atmospheres possible. The Kepler spacecraft was particularly successful in this respect proving that planets are no exception, but rule around stars in the Milky Way galaxy. The follow-on investigation of the best candidate planets by giant space and terrestrial telescopes became a promising field of search after both life signatures and signatures of a technical civilization in these extrasolar planetary systems. Consequently the methodology of astrobiology and SETI is converging in recent years. SETI is changing in many respect, "old SETI" develops into a "new SETI", which has more projects in common with astrobiology than ever before. Based on the SETI literature this paper is trying to make this process known in detail.

Motto: “... look for what's detectable, not for what's probable."

Freeman Dyson

\section{Old SETI}

\subsection{Orthodox SETI - the first decades}

CETI (Communication with Extraterrestrial Intelligence) has started as a new discipline in 1959 after the publication in Nature of the seminal paper of Cocconi and Morrison "Searching for Interstellar Communication" [1]. They have argued that existing radio telescopes were capable of receiving purported artificial messages from advanced civilizations far beyond our Solar System and outlined a strategy which is the cornerstone of microwave SETI up-till-today. The basic assumptions of this strategy are the following:

$1 /$ connection by electromagnetic waves with distant technical civilizations is much easier, cheaper and faster than interstellar travel;

$2 /$ radio is the most suitable technique for long distance communication;

3 / the microwave spectrum around $1-10 \mathrm{GHz}$ is favored because natural cosmic background noise is minimal;

4/ the selected frequency should be near to the well known hydrogen emission line at 21 $\mathrm{cm}$ wavelength, because of the conspicuous character of this important line in radio radiation of the universe (water hole). 
Practically the same assumptions were used in 1960 when Frank Drake (independently from Cocconi and Morrison) started his OZMA Project at the National Radio Astronomy Observatory in Green Bank. He observed two near-by solar-type stars for a very limited period with an 85 foot radio telescope. Several dozen radio SETI searches followed mostly in the US and in the Soviet Union based practically on the same assumptions.

Since there was no proposal in this strategy on the best stellar candidates to be investigated, two concurrent ideas have emerged: either to point towards selected near-by solar type, single stars, or to scan the entire sky expecting that somewhere an interesting signal will appear. Famous targeted SETI searches were initiated by the Harvard University: the META (Megachannel Extraterrestrial Assay) project, which later progressed into BETA registering the target radiation on almost one billion narrow channels. The method has been applied also in Argentina and in other radio observatories. An equally important initiative was the NASA program in Arecibo which culminated in 1992 by starting a targeted search called Microwave Observing Project (later High Resolution Microwave Survey). Their Multi-Channel Spectrum Analyzer proved to be an equally efficient tool in searching narrow signals in the microwave spectrum.

But just one year later, as a consequence of the veto of the Congress, it was forbidden for NASA to continue the financial support of the HRMS program. The all sky survey project at Goldstone has been interrupted, but the privately funded targeted search continued as the Phoenix Project of the new SETI Institute. In the following years it has made shorter observation campaigns on different radio telescopes in the US, Australia, France, UK etc. The longest observation series has been carried out at the Ohio State University from 1973 until 1997 by the 176 foot "Big Ear" telescope. Their most famous result was the detection of the so called "Wow! Signal" in 1977. It was an intensive narrow-band signal, but has been recorded only once by a single instrument.

Another promising US university program has been started in the mid-70s at Berkeley. The SERENDIP (Search for Extraterrestrial Radio Emission from Nearby Developed Intelligent Population) is also an all-sky survey, but in a piggy-back mode on the Arecibo telescope. It has no influence on the radio astronomical program of the telescope, but collects through its own instrument an enormous quantity of measured data from randomly selected targets. In order to facilitate data reduction and interpretation SETI@home, an innovative method to mobilize millions of volunteers with free PC capacities all over the world, has been initiated in 1999. In a few years an enormous international network of computers has been formed to improve data analysis. This great success has proved that the public is ready to help SETI, if there is such an opportunity.

\subsection{Signal analysis}

As it is obvious signal analysis was and is a serious technical problem in orthodox microwave SETI. A challenge facing all these projects was differentiation between signal and the ever-present natural background of cosmic noise. According to Paul Shuch [2] "Whereas natural thermal emissions are broadband, extending across the entire electromagnetic spectrum, it is expected that signals used for deliberate electronic communication over interstellar distances will have narrow-band components. ... Assuming a sufficiently narrowband receiver, there are billions of possible frequencies to which it might be tuned. Here is where digital signal 
processing (DSP) techniques become an important part of SETI research. It is common in SETI practice to receive, amplify, filter and digitize extremely wide portions of the electromagnetic spectrum. DSP is then employed to subdivide the broad received spectrum into a multitude of contiguous, vanishingly narrow frequency bins, each of which excludes much of the broad spectrum of noise ratio. Most frequently the FFT is employed to produce these narrow bins. ... A limitation of the FFT is that it is optimized for the detection of sinusoidal signal components. If the nature of the incoming signal is unknown, one would desire an adaptive transform to detect it. One such tool, which makes no a priori assumptions as to the characteristics of the signal hidden in the noise, is the Karhunen-Leove transform (KLT). ... Unfortunately the KLT is extremely demanding of computer power."

The procedure to detect unambiguously a radio message or signal from an extraterrestrial technical civilization (ETC) is a very complicated one. Although seemingly there was a consensus among SETI participants in the first decades to follow the strategy and philosophy of the founders of SETI, the practical situation proved to be more complicated than previously thought. According to Lemarchand [3], who has defined a "space of communication" for an interstellar communication channel, there are many decision steps in orthodox microwave SETI until the information content of a message can be interpreted:

direction $\rightarrow$ timing, synchronicity $\rightarrow$ frequency $\rightarrow$ transmitting output power $\rightarrow$ polarization $\rightarrow$ modulation type $\rightarrow$ signal bandwidth $\rightarrow$ signal rate $\rightarrow$ code $\rightarrow$ semantics $\rightarrow$ kind of information content

As summarized by John Billingham in his "Decision Diagram for SETI" [4] orthodox SETI researches should come to at least a dozen important decisions when initiating a project of observations. We are confined only to the right side of the original figure, where the orthodox SETI program decisions are illustrated from the selection of an electromagnetic carrier to signal processing:

\footnotetext{
$\mathrm{EM} \rightarrow$ microwave $\rightarrow$ receive $\rightarrow$ beacon $\rightarrow$ pulse $\rightarrow$ sequential $\rightarrow$ many antennas $\rightarrow$ coherent $\rightarrow$ many beams $\rightarrow$ signal processing
}

In spite of these difficulties and of some alternative ideas and experiments "orthodox SETI" remained the dominant paradigm up-till-now. As a typical example we refer to the "Call for papers" of the 5th IAA Search for Life Signatures Symposium, March 20-21, 2014 Paris, France, where the definition of SETI is given as follows:

"SETI (the "Search for Extraterrestrial Intelligence") refers to the experiments intended to find either radio or optical signals from extraterrestrial societies situated on planets around other stars. ... These attempts to detect extraterrestrial signals are called 'Passive SETI'."

The "motto" introducing this chapter characterizes the dominant SETI strategy of this period: “...look for what's detectable, not for what's probable." Nevertheless from the very beginning some independent thinking scientists have proposed alternative methods and strategies, but without imminent result. 


\subsection{Some unorthodox SETI projects}

Among the unorthodox SETI projects the first and most wide-spread is optical SETI. Already in 1961 Robert Schwartz and Charles Townes have suggested in a Nature paper [5] to use optical maser for interstellar communication. They have proposed to use optical telescopes to discover unusually short (microseconds) pulses in stellar brightness. The first Optical SETI Observatory, however, started to work only in 1995 in Columbus, Ohio. Other experiments have followed later, when laser technology has developed into an excellent communication tool. As one of the pioneers of optical SETI, Monte Ross has emphasized in his book some advantages of optical SETI over microwave SETI: "The development of laser communication for submarines, aircraft and military satellites proved that short bursts of laser light are more efficient than continuous waves at carrying information. Although each pulse has a high peak power, the laser is inactive for most of the time and therefore has a low total consumption. With transmissions in brief bursts, each pulse could readily outshine any star in the field of view of the collector. Whatismore, the shorter the pulse, the less background light there is per pulse to compete with the signal. Reducing the pulse to nanosecond intervals makes any signal detected even more obviously of artificial origin, as such short flashes are unlikely to occur naturally." [6] Today there are serious efforts to develop an all-sky optical SETI at the Harvard Observatory. Optical SETI was or is carried out also at University of California, Berkeley, as well as in Russia, Australia and Argentina.

Some popular unorthodox, but only symbolic SETI experiments have been carried out in the 70s: the Arecibo Message sent to globular cluster M13 in 1974, the two plaques on Pioneer 10 and 11 (launched in 1972 and 1973), and the Voyager Golden Records launched in 1977 on Voyager 1 and 2 with messages of mankind.

A common, very complicated problem is, however, how to decide that a recorded signal is of artificial origin. The opinion of Paul Shuch [2] is that "such signals must exhibit some reasonable combination of the following hallmarks of artificiality:

- spatial/temporal characteristics consistent with sidereal motion;

- coherence not achievable by known natural emission mechanism;

- Doppler signatures indicative of planetary motion;

- frequency selection which exhibits a knowledge of one or more universal constants; and information content suggestive of a mathematically based culture."

"The common denominator of all these characteristics, in fact of all human (and we anticipate, alien) existence, is that they are anti-entropic. Any emission which appears (at least in the short term) to defy entropy is a likely candidate for an intelligently generated artifact. In that regard, periodicity is a necessary, though not a sufficient condition for artificiality."

\section{Critisism}

It is absolutely understandable that after so many unsuccessful attempts to detect any messages of an extraterrestrial technical civilization, voices of criticism appeared in different forms everywhere. According to Milan Ćircović [7] there are two groups of "skeptics": the fundamentalists and the instrumentalists. He has formulated it this way:

"There are at least two entirely different meanings of skepticism:

1/ a fundamental rejection of the existence of ETI and/or communication-capable beings (independent from methodology) and 
2/ deals mainly with "instruments" i.e. practices and procedures of real, human-conducted research (does not touch upon existence or density of inhabited planets)."

Ćircović argues that a delineation of various forms of skepticism is an important task. The skepticism towards SETI has already been outstandingly influential in determining the fate of some important SETI projects, like NASA HRMS. It has not been highlighted enough that it is a perfectly coherent position that life and intelligence are frequent in the Galaxy, but the recognition of them is difficult or impossible. Is a person holding such beliefs opponent or supporter of SETI? - asks Ćircović.

\subsection{Fundamentalist skepticism}

According to Ćirković all classical anti-SETI books and papers of fundamentalist skeptics like Viewing, Hart, Webb, Tipler, Barrow, Mayr, Ward \& Brownlee ("Rare Earth"), Gibson ("The Reason Why...") and others are based on one or more of the following three simple arguments :

1/ Fermi paradox (1950)

"...if any ETC in the Galaxy developed interstellar travel, it would have easy - given the amount of time provided by the age of our stellar system - either colonized all planetary systems in the Galaxy or at least have detectable traces and manifestations..." [8]

Progress in astrobiology on the one side and Kepler results on the other have actually made it even more disturbing in recent years.

2/ Simpson's argument (1964)

"...the probability of anything even remotely similar to humans evolving on another planet is minuscule, since biological evolution is dominated by contingency..."

"...the chance for encountering another technical civilization elsewhere in the Galaxy at this particular epoch is negligible." [9]

3/ Carter's argument (1983)

The problem is "our ignorance about the characteristic temporal scales of biological evolution leading to emergence of intelligent observers." "The existence of other intelligent observers in the Galaxy at present is improbable" because "we are the first biosphere which has completed the entire sequence of steps leading to intelligent observers." [10]

What kinds of steps are critical? Robert Hanson gave a concrete list in 1998: "The development of intelligent life requires many (4 to 7) steps, such as the emergence of singlecelled life and the transition from unicellular to multicellular life forms. Since we have not observed intelligent life beyond our planet, there seems to be a development step that is so difficult and unlikely that it 'filters out' nearly all civilizations before they can reach a spacefaring stage."[11] This idea is called nowadays "The Great Filter".

The consequence of fundamental skepticism is that SETI targets don't exist, neither in our Galaxy nor in the whole Universe. According to Ćircović, however, this consequence is based always on arguments where additional specifications are needed [7]. E.g. in "rare Earth" argumentation "extraterrestrial complex life, including all conceivable intelligent life, is incredibly rare due to the lack of one or more rare ingredients playing a role in the history of our planet." Another important point is that fundamental skepticism denies the existence of any form of continuity between ETI and less complex forms of organization of matter. 
It is certainly a weak, but popular argument against any further search that the searches uptill-now did not produce any convincing result. As it will be demonstrated later, there are many reasonable suggestions how to carry out "new SETI" which is different from the orthodox old one.

\subsection{Instrumentalist skepticism}

According to Ćirković [7] "there is no necessity to assume the 'rare Earth' or any other theoretical construction to explain the negative result of ... SETI projects so far." "...evolution in each individual biosphere is a perfect natural segment of the overall astrobiological evolution ... so we need not accept any form of terrestrial or human exceptionalism."

He would like to change SETI methodology and SETI philosophy radically urging that the search should concentrate on artifacts and technosignatures; take postbiological evolution and the possibility of a superintelligent ETC into consideration; aim at an expansion of the list of admissible SETI targets; and launch a tighter interdisciplinary cooperation.

Already Steven Dick [12] raised the problem that it is only a prejudice that intelligent extraterrestrials are necessarily biological entities - neglecting the very real possibility of a postbiological evolution. This possibility changes the fundamental assumption that SETI targets should be habitable exoplanets and degrades further the weekly motivated concept of "habitable zones". (See also [13]).

Several recent authors belong to representatives of instrumentalist skepticism, like Ćirković \& Klaes in “Has SETI Been Barking Up the Wrong Tree (Mostly)?”[14], William Edmondson in "Targets and SETI" [15], Richard Carrigan in "Is interstellar archeology possible?" [16] and Paul Davies in his book "The Eerie Silence" [17].

Their common conviction is that some alternative approaches to orthodox SETI are a necessity independently from the assumption that there are many or only a very few intelligent civilizations in our Galaxy.

Motto: "The only way of discovering the limits of possible is to venture a little way past them into the impossible."

Arthur Clarke

\section{New SETI}

As one of the leading scientists behind old SETI, Jill Tarter has declared to Space Daily in 2013: "Exoplanets and extremophiles have been real game changers ... so there might be a lot more habitable real estate out there than we once thought. We are now willing to re-question everything and think about what we can do now that was not considered possible when we got started."

\subsection{Outside our Solar System}

This is without any doubt a paradigm shift. Due to the new technical challenges SETI has new possibilities in extrasolar planetary systems and also in far away galaxies. First of all the frequency range interesting for any SETI experiment has been selected in the early 1960s, when telescopes existed only on the surface of the Earth. It was implicitly supposed that any communication with extraterrestrials will be possible only through the spectral "windows" of the terrestrial atmosphere (microwave and optical windows). Nowadays the situation has 
changed. On the one hand the increased terrestrial noise background is a serious source of worry just in these wavelength bands, on the other many sensitive astronomical satellites are functioning outside the Earth's atmosphere. Although there is no astronomical observatory on the far side of the Moon yet, but new space probes equipped with very sensitive sensors work in outer space almost continuously in frequency bands unattainable from the surface of the Earth. Although electromagnetic waves are still preferred in current SETI programs, but a systematic investigation to evaluate all possible information carriers (from and to interstellar distances) has been also initiated.

According to Lemarchand [18] the possible information carriers are the following:

a/ Boson astronomy:

photons (radio, IR, optical, UV, X, gamma rays); gravitons (gravity waves)

b/ Particle astronomy:

microscopic particles

fermions (neutrinos, electrons and protons - cosmic rays), heavy particles (atomic nuclei - cosmic rays);

macroscopic particles or objects (meteorites, dust, asteroids, comets).

c/ Direct techniques:

space probes, exploration, astroengineering activity in the Solar System

$\mathrm{d} /$ Unknown information carrier

e.g. DNS or genetic code

Only a few of these potential information carriers have been studied up-till-now and only sporadic SETI efforts have been made. The first experiment outside the microwave and the optical window was attempted in the UV region in 1974 using the Copernicus satellite. The search went on after spectral lines of laser origin near the Lyman $\alpha$ line at $1215 \AA$ in the spectrum of three stars.

In the infrared region Dyson has suggested already in 1960 to search after infrared excess in stellar spectra. He emphasized the possibility that very advanced (Kardashev II type) civilizations might use the total energy of their central star by building a sphere around the system. The "Dyson spheres" should be exceptionally bright objects in the infrared. In the early 90s the data base of the IRAS infrared satellite has been investigated in Japan looking for infrared excess objects. The next infrared satellite, ISO, had already a short SETI observation project looking after Dyson spheres around 8 stars.

In the gamma-ray region a search was carried out using the data base of the Compton GRO satellite. The idea was to look after strong penetrating gamma-rays created by bulk antimatter burning used by an alien advanced technology. Strange gamma-ray bursts are also frequent candidates for a high technology which can modify or influence the energy output of a star.

As far as it is known there are no attempts yet to discover ET messages in any other possible radiation form neither in boson nor in particle astronomy.

Richard Carrigan [16] has summarized the new possibilities to discover traces of a very advanced technical civilization somewhere outside our solar system - without waiting for any signals or messages from them! He calls this type of activity "interstellar archeology (IA)". He 
emphasizes that IA is the search for large scale signatures of civilizations beyond the solar system - whether still existing or not. "Detection of a cultural signal would be in the spirit of IA just an artifact like pottery or garbage dumps give messages from the past through archeology. Most of these archeological signatures on Earth were not intended as messages to the future. They did not require a conscious decision to try to communicate."

Among the possibilities first of all the new perspectives of direct observations of exoplanets should be mentioned. Astronomers already started to study the spectrum and composition of exoplanetary atmospheres and have discovered methane, water, carbon monoxide and carbon dioxide in some of them. These are important life signatures, but also cultural based signatures can be expected? - asked Carrigan. One possibility is a spectral line due to radioactive "salting" of a star to introduce short-lived nuclear species with a strong optical resonant absorption line. Drake and Shklovskii have suggested searching after technetium, an element with no stable isotope. Another possibility is (according to Whitmire and Wright) to use a star as a disposal site for nuclear waste. Getting a recognizable signal through plutonium would require all of a civilizations fissile output.

Carrigan mentioned some other possibilities as well, e.g. manipulating a star before it enters the red giant phase (blue stragglers), or looking after SETI signals or any other sign of heightened astroengineering activity when the central star is already near to the end of its main sequence life.

Although today there is no real possibility yet to observe directly the surface of any exoplanets, but the progress in this direction is very fast. It is not difficult to imagine giant space telescopes taking photos of the disc of planets of relatively near-by stars. If the resolution of the images improves we can hope to be able to decide for instance whether there is any artificial illumination on the dark side of an Earth-like planet or moon. Supposing that there is a largescale astroengineering activity in a relatively near-by planetary system one can try to observe transits before the stellar disk of strange objects (probably of artificial origin). Any sign or traces of technology ("technomarkers") would be a giant step in SETI.

But it is necessary to make it unmistakably clear that at present nobody has observed a real phenomenon on astrophysical objects, which arguably hints at the intervention of an advanced technology.

\subsection{Within our Solar System}

One can search after technomarkers within the Solar System as well. Many space probes follow with attention the Sun, Moon, planets, satellites, asteroids and comets. The surfaces of some of these planetary bodies (the Moon and Mars in particular) are already littered with remnants of terrestrial structures, like space probes, rovers, rocket stages etc. This will make a distinction between traces of terrestrial and extraterrestrial technology in the future quite difficult. Nevertheless Davies \& Wagner [19] has initiated a search after artifacts of extraterrestrial origin on the surface of the Moon using high-resolution images of lunar satellites. Robert Freitas \& Francisco Valdes [20] have carried out searches after artificial objects near the Earth-Moon Lagrangian points, but in vain. The asteroid belts with many thousands of unknown celestial bodies are also promising places for a direct SETI investigation - as proposed by Michael Papagiannis [21]. 
There are and were several efforts to prove that traces of an alien technological civilization are still present on the surface of the Earth (Nasca lines, pyramids etc.), but without any convincing evidence. Other authors following an idea raised in the early sixties looked into the genome to find traces of a directed panspermia. In some recent papers Vladimir Shcherbak \& Maxim Matukov [22] have claimed that studying the terrestrial genetic code it displays a thorough precision-type orderliness matching the criteria to be considered an informational signal. The code reveals an ensemble of arithmetical and ideographical patterns of the same symbolic language.

\subsection{Some new telescopes planned or realized}

First of all the Allen Telescope Array (ATA) of the SETI Institute and the University of California should be mentioned. Initial funds were given by Paul Allen. According to the original plans 350 offset-Gregorian $6.1 \mathrm{~m}$ radio dishes should have been built and connected for multibeaming (in which DSP technology is used to look in multiple directions on the sky) simultaneously carrying out radio astronomical and SETI observations (targeted search). In 200742 antennas were ready, but the work had to be stopped because of lack of funding. Upgrades of these telescopes from 1-8 GHz to 1-18 GHz frequency range has started in 2012.

The Low-Frequency Array (LOFAR) built between 2006 and 2012 is the largest connected radio telescope of the world. It operates with 25000 omni-directional small antennas using an aperture synthesis technique at radio frequencies below $250 \mathrm{MHz}$. The total collecting area in the Netherlands, Germany, UK, France and Sweden is up to $1 \mathrm{~km}^{2}$. It is capable to survey the Universe at $10-240 \mathrm{MHz}$ with greater resolution and sensitivity than previously. Its use for SETI is also contemplated.

The Square Kilometer Array (SKA) is a very ambitious radio astronomical project, which will be realized in South Africa. The frequency range will be from 0.15 up to $20 \mathrm{GHz}$, the field of view 1 square degree at $1.4 \mathrm{GHz}$, the number of instantaneous beams at least 100 , the angular resolution $<0.1 \operatorname{arcsec}$ at $1.4 \mathrm{GHz}$.

In the optical range the Colossus telescope is representing the next promising step. It will be an infrared telescope with a primary mirror of $77 \mathrm{~m}$, composed from sixty very thin $8 \mathrm{~m}$ mirror segments. A huge collecting area and an adaptive optics system to correct for atmospheric effects are planned. The field of view will be only a few arcseconds. Since finding a civilization through its waste heat radiation will be possible, the Colossus might be a tool to survey potential technical civilizations out to at least $60 \mathrm{ly}$. It will search also after distinctive transient events ("ping detection").

\subsection{Paper projects}

There are some interesting paper projects worth mentioning.

Claudio Maccone [23] has suggested that advanced technical civilizations use their own star, or a black hole, or a gigantic black hole in the center of their galaxy as a gravitational lens in interstellar or even intergalactic communication.

The Benfords [24] have suggested that pulsed, narrowly directed, broadband blips may come from the galactic center in order to draw the attention of emerging new civilizations. Our present instruments are not capable to observe such a phenomenon ("Benford beacons").

Clément Vidal [25] has argued that black holes can be attractors for very advanced civilizations which are capable to utilize their enormous gravity. 


\section{Active SETI or METI}

The first Message to Extraterrestrial Intelligence (METI) initiated by Frank Drake in 1974 has already been mentioned. Influenced by the negative reaction of the science community there was no follow-on experiment for 25 years. When in 1999 Aleksandr Zaitsev [26] and his group started to send messages (on a commercial basis) towards selected stars through the Yevpatoria radar the reaction was mixed. After a long discussion period the IAA SETI Committee is still divided whether sending messages towards the stars is acceptable or not. The Royal Society has organized a workshop in 2010 inviting both sides with moderated discussions on the topic.

Let us quote the opinion of some respected scientists and celebrities! Among the supporters Vitaly Ginzburg wrote already in 1973 that “...there is nothing more dangerous than to speak about the danger of communication." On the other side, Eric Chaisson declared that "Advanced life, anywhere in the cosmos, will tend to control other life", therefore any message or signal sent to an ETC to draw its attention might be a danger for mankind. Stephen Hawking is also convinced that "We only have to look at ourselves to see how intelligent life might develop into something we wouldn't want to meet." (BBC News 25 April 2010) Today nobody can tell how risky a radio contact with an alien civilization might be, but it is obvious that there is some kind of risk. There is a good summary of the topic "The benefits and harm of transmitting into space" by Jacob Haqq-Misra et al. [27].

My opinion is that METI or active SETI (both names are used) is not belonging to SETI at all, since it is not a scientific search. And it is important to mention that carrying out any kind of passive SETI for a long time and covering the whole sky is economically feasible and also can provide new results for astronomy or other sciences. Active SETI is not in such a position.

\section{Conclusions}

After 55 years the history of SETI research has already a perspective. American scientists and amateurs played always a dominant role, although Soviet-American cooperation in this field was important for a long time. Some individuals of other countries have also contributed significantly. First the paper project Cyclops was the symbol of SETI efforts, later almost all activity has concentrated on the NASA project (targeted and all sky search in the microwave window). After 1993 the dominant American effort was Phoenix organized by the SETI Institute. Finally "orthodox SETI" and almost all professional SETI researchers concentrated their efforts on establishing ATA, which is still in an uncertain position.

Meanwhile the failure of half a century of SETI research called different new competing projects into existence. The diversity and broadening of the spectrum of SETI ideas and projects is certainly an advantage, but many of them suffer from a weak financial basis and a lack of professional staff. Therefore "new SETI" is a promise for the future, but not really convincing as an alternative for "old SETI" at present.

\section{Acknowledgements}

The author thanks the organizers of the Mondello Workshop for their kind invitation and the Konkoly Observatory of the Hungarian Academy of Sciences for some financial support. Also thanks to Stelio Montebugnoli for his opinion as a referee of the paper. 


\section{References}

[1] G.Cocconi, P. Morrison, Searching for interstellar communication, Nature, 184, 8441959.

[2] P. Shuch, P, A half-century of SETI science, in Searching for Extraterrestrial Intelligence: SETI Past, Present, and Future, P. Shuch (ed.) Springer-Praxis, Chichester, 2011.

[3] G. Lemarchand, Detectability of extraterrestrial technological activities SETIQuest 1, 31992.

[4] J. Billingham, SETI and society - decision trees, Acta Astronautica 51, 667. 2002.

[5] R. Schwartz, C. Townes, Interstellar and interplanetary communication by optical maser, Nature $\mathbf{1 9 0}$, 205. 1961.

[6] M. Ross, The search for extraterrestrials - intercepting alien signals, Springer-Praxis, Chichester UK p.111. 2009.

[7] M. Ćirković, Who are the SETI skeptics? Acta Astronautica 89, 38. 2013.

[8] S. Armstrong, A. Sandberg, Eternity in six hours: intergalactic spreading of intelligent life and sharpening the Fermi paradox, Acta Astronautica 89, 1, 2013.

[9] G. Simpson, The nonprevalance of humanoids, Science, 143, 769. 1964.

[10] B. Carter, The anthropic principle and its implications for biological evolution, Philos. Trans. R. Soc. London A 310, 347. 1983.

[11] R. Hanson, The Great Filter - are we almost past it? http://hanson.gmu.edu/greatfilter.html. 1998.

[12] S. Dick, The postbiological universe and our future in space, Futures 41, 578, 2009.

[13] I. Almár, SETI terminology - do we interpret SETI terms correctly? Acta Astronautica, 68, 351. 2011.

[14] M. Ćircović, L. Klaes, Has SETI been barking up the wrong tree (mostly) Space Daily Aug. 7, 2006.

[15] W. Edmondson, Targets and SETI: shared motivation, life signatures and asymmetric SETI, Acta Astronautica doi: 10.1016/j.actaastro.2010.01.017. 2010.

[16] R. Carrigan, Is interstellar archeology possible? Acta Astronautica 78,121 2012.

[17] P. Davies, The Eerie Silence, Allen Lane, London 2010.

[18] G. Lemarchand, Counting on beauty: the role of aesthetic, ethical and physical universal principles for interstellar communication, in The Art and Science of Interstellar Message Composition, D. Vakoch (ed.) MIT Press, Cambridge MA 2006.

[19] P. Davies, R. Wagner, Searching for alien artifacts on the moon, Acta Astronautica doi: 10.1016/j.actaastro.2011.10.022. 2011.

[20] R. Freitas, F.Valdes, A search for natural or artificial objects located at the Earth-Moon libration points, Icarus 42, 442, 1980.

[21] M. Papagiannis, Natural selection of stellar civilizations by the limits of growth, Q.J.R.Astro.Soc., 25, 309. 1984. 
[22] V. Shcherbak, M. Makukov, The "Wow Signal” of the terrestrial genetic code Icarus 224, 228. 2013.

[23] C. Maccone, SETI among galaxies by virtue of black holes, Acta Astronautica 78, 109. 2012.

[24] J. Benford, G. Benford and D. Benford, Searching for cost-optimized interstellar beacons,

Astrobiology 10, 491, 2010.

[25] C. Vidal, Black holes: attractors for intelligence, preprint arXiv: 1104.4362. 2011.

[26] A. Zaitsev, Sending and searching for interstellar messages, Acta Astronautica 63, 614. 2008.

[27] J. Haqq-Misra, M. Busch, S. Som and S.Baum, The benefits and harm of transmitting into space, Space Policy 29, 40. 2013.

\section{DISCUSSION}

VLADIMIR LIPUNOV: I am very wondered why you do not include Shklovsky's later idea about short time of the civilization. From my point of view the more important Tsiolkovsky paradox for Grand Universe which has infinitely attempts to create intelligent life.

IVÁN ALMÁR: It is certainly true that Prof. Shklovsky was not only one of the pioneers of SETI (see his book which was translated into English with Carl Sagan as coauthor), but also one of the first scientists emphasizing the importance that "there are no miracles on the sky". In the latest years of his life he really became the first "skeptic" with respect to SETI.

Yes, Konstantin Tsiolkovsky has really written important philosophical papers on the problem, which became later known as "Fermi paradox", but this part of his activity was almost unknown in other countries. The traditional name of this important problem remained "Fermi paradox" in spite of the fact that later several authors contributed significantly to the topic (Viewing, Hart, Tipler).

JIM BEALL: I don't advocate this but some of my colleagues believe we are here in a guarded preserve: not contacted but being studied. Can you comment on this?

IVÁN ALMÁR: It is possible, but certainly improbable that members of an ETC travel interstellar distances to study the population of the Earth without trying to contact us. If UFO observations were reliable and would produce some artifact then in order to decide whether it is artificial and of extraterrestrial origin the science community should investigate it with all possible means. There is, however, no real progress in the "UFO observations" themselves - although several new and effective technical means (like smart phones or video cameras) are available almost everywhere. 